\title{
How Do Drosophila Stay Awake during the Daytime? Dopaminergic Neurons Are Inhibited by the Pigment Dispersing Factor Signaling Pathway to Promote Wakefulness
}

\author{
Rosalind S.E. Carney, DPhil
}

Sleep is regulated by the circadian clock so that organisms can best function with respect to their local environment. Significant insight into circadian clock circuitry and associated neurotransmitter functions have enabled the development of treatments for individuals who have difficulties with sleep onset, sleep maintenance, or arousal from sleep. However, less is currently known about the neural mechanisms that maintain wakefulness during the daytime. Narcolepsy, a chronic sleep disorder characterized by excessive daytime sleepiness, affects around one in every 2000 people in the United States (U.S. National Library of Medicine, 2018). Narcoleptics can have difficulty adhering to conventional working hours and maintaining societal interactions. Therefore, it is important to ask what are the neural mechanisms that promote the maintenance of wakefulness during the daytime.

Drosophila melanogaster is a useful model for this question because they have regular sleep bouts during the daytime and exhibit sleep behavior similarities to mammals (Hendricks et al., 2000; Shaw et al., 2000). Sleep behavior can be monitored by placing Drosophila in glass tubes in which activity is recorded by infrared beam. Pigment dispersing factor (PDF) is a circadian neuropeptide that is known to promote wakefulness in Drosophila. In their eNeuro publication, Potdar and Vasu (2018) performed a wide array of experiments in Drosophila to identify the downstream targets and signaling interactions of the PDF receptor (PDFR).

A prior study in Drosophila showed that $p d f r^{5304}$ and pdfr $^{3369}$ loss-of-function mutations increased sleep during the daytime and nighttime (Chung et al., 2009). When

\footnotetext{
Received September 4, 2018; accepted September 4, 2018; First published September 11, 2018.

DOI:http://dx.doi.org/10.1523/ENEURO.0348-18.2018
}

This is an open-access article distributed under the terms of the Creative Commons Attribution 4.0 International license, which permits unrestricted use, distribution and reproduction in any medium provided that the original work is properly attributed.
Potdar and Vasu backcrossed the $p d f r^{5304}$ and $p d f r^{3369}$ mutants over seven or eight generations to the Iso31 $\left(w^{1118}\right)$ background, they found that sleep was increased during the daytime only. This increase in daytime sleep was replicated during the subjective daytime when the pdfr mutants were transferred to constant darkness. Furthermore, compared to controls, both pdfr mutants also fell asleep sooner and slept longer within a typical daytime sleep bout, although the number of total sleep bouts was similar in all groups. Taken together, these observations indicate that in the absence of functional PDFR, daytime sleep is initiated sooner, is more consolidated, and is of longer duration.

Potdar and Vasu next altered pdfr expression in 26 GAL4 lines representing distinct neuronal subsets that included circadian pacemakers, higher-order processing centers, or neuronal groups based on neurotransmitter expression. However, sleep was altered consistently in only a few of these lines. From these data, Potdar and Vasu hypothesized that circadian clock neurons may not be the downstream targets of the PDFR signaling pathway that promotes wakefulness during the daytime. However, further analyses showed that decreasing PDFR signaling to dopaminergic neurons increased day-time sleep, whereas increasing PDFR signaling to dopaminergic neurons suppressed day-time sleep and made it fragmented, in addition to delaying sleep onset. These observations suggest that dopaminergic neurons are the downstream targets of the PDFR signaling pathway that initiates and maintains wakefulness during the daytime.

A prior study had shown that dopamine acts on large ventral lateral neurons (I-LNv) to promote wakefulness (Shang et al., 2011). To determine whether PDF+ neurons synapse with dopaminergic axons, Potdar and Vasu used a GFP reconstitution across synaptic partners (GRASP) experiment. GRASP is a system in which two complementary GFP fragments, expressed on different cells, reconstitute as a GFP florescence reporter only at the site 

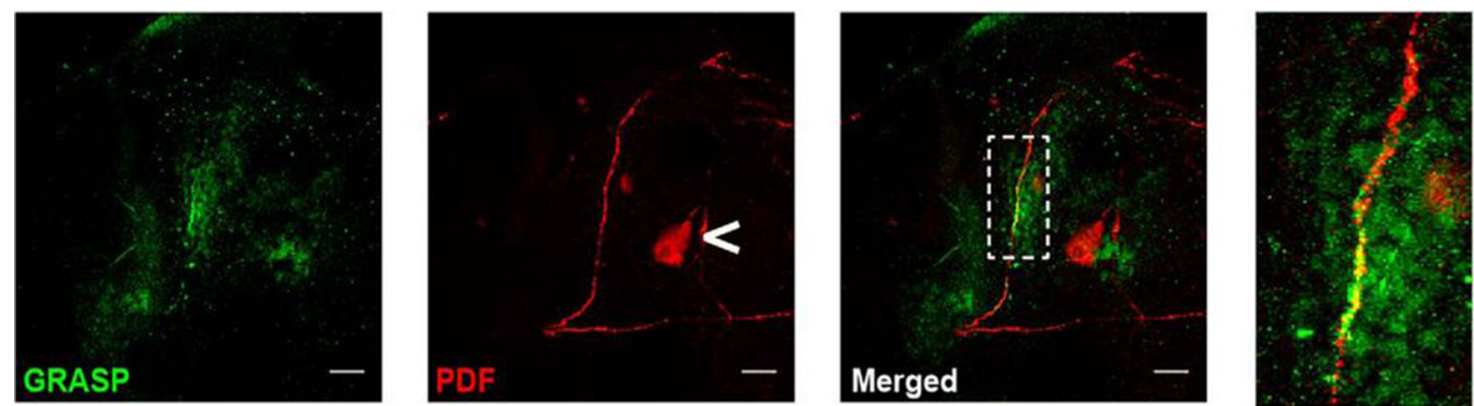

Figure 1. Anatomic connections between PDF+ neurons and dopaminergic neurons. GRASP signal (green) colocalized with the ascending portion of $\mathrm{s}-\mathrm{LNv}$ dorsal projections labeled with an antibody against PDF (red).

of a synapse (Feinberg et al., 2008). When the two GFP fragments were expressed in PDF + and dopaminergic neurons, staining with an anti-GFP antibody revealed colocalization in the ascending portion of small lateral ventral neuron (s-LNv) dorsal projections, confirming synaptic connections (Fig. 1). s-LNv have been proposed to have an auxiliary role in the wake-promoting effects of I-LNv (Parisky et al., 2008; Potdar and Sheeba, 2012). Therefore, Potdar and Vasu examined the effects on sleep when a neurodegenerative protein was used to selectively render s-LNv dysfunctional (Sheeba et al., 2010), while simultaneously changing the electrical properties of I-LNv (Nitabach et al., 2006). These results validated that I-LNv modulate wakefulness and further show that functional $\mathrm{s}-\mathrm{LNv}$ are required to mediate these effects.

In addition, by measuring calcium levels as indicators of neuronal activity, Potdar and Vasu showed that dopaminergic neurons, the likely targets of PDFR signaling, are less active during daytime. As PDF levels are high during the daytime, it is likely that the dopaminergic neurons that mediate wakefulness are, in fact, sleep-promoting and inhibited by PDF. These findings have identified a circadian clock pathway that promotes wakefulness during the daytime in Drosophila.

\section{References}

Chung BY, Kilman VL, Keath JR, Pitman JL, Allada R (2009) The GABA(A) receptor RDL acts in peptidergic PDF neurons to promote sleep in Drosophila. Curr Biol 19:386-390. CrossRef Medline Feinberg EH, Vanhoven MK, Bendesky A, Wang G, Fetter RD, Shen $\mathrm{K}$, Bargmann $\mathrm{Cl}$ (2008) GFP reconstitution across synaptic part- ners (GRASP) defines cell contacts and synapses in living nervous systems. Neuron 57:353-363. CrossRef

Hendricks JC, Finn SM, Panckeri KA, Chavkin J, Williams JA, Sehgal A, Pack Al (2000) Rest in Drosophila is a sleep-like state. Neuron 25:129-138.

Nitabach MN, Wu Y, Sheeba V, Lemon WC, Strumbos J, Zelensky PK, White BH, Holmes TC (2006) Electrical hyperexcitation of lateral ventral pacemaker neurons desynchronizes downstream circadian oscillators in the fly circadian circuit and induces multiple behavioral periods. J Neurosci 26:479-489.

Parisky KM, Agosto J, Pulver SR, Shang Y, Kuklin E, Hodge JJ, Kang K, Liu X, Garrity PA, Rosbash M, Griffith LC (2008) PDF cells are a GABA-responsive wake-promoting component of the Drosophila sleep circuit. Neuron 60:672-682. CrossRef

Potdar S, Sheeba V (2012) Large ventral lateral neurons determine the phase of evening activity peak across photoperiods in Drosophila melanogaster. J Biol Rhythms 27:267-279. CrossRef Medline

Potdar S, Sheeba V (2018) Wakefulness Is Promoted during Day Time by PDFR Signalling to Dopaminergic Neurons in Drosophila melanogaster. eNeuro 5:ENEURO.0129-18.2018.

Shang Y, Haynes P, Pírez N, Harrington KI, Guo F, Pollack J, Hong P, Griffith LC, Rosbash M (2011) Imaging analysis of clock neurons reveals light buffers the wake-promoting effect of dopamine. Nat Neurosci 14:889-895. CrossRef

Shaw PJ, Cirelli C, Greenspan RJ, Tononi G (2000) Correlates of sleep and waking in Drosophila melanogaster. Science 287:18341837.

Sheeba V, Fogle KJ, Holmes TC (2010) Persistence of morning anticipation behavior and high amplitude morning startle response following functional loss of small ventral lateral neurons in Drosophila. PLoS One 5:e11628. CrossRef

U.S. National Library of Medicine (2018) Narcolepsy. Available at https://ghr.nlm.nih.gov/condition/narcolepsy\#statistics. Published August 21, 2018. 